\title{
A Double scattering polarimeter for the P2 experiment at MESA
}

\author{
Matthias Molitor*† \\ Institut für Kernphysik, Johannes Gutenberg-Universität Mainz, Germany \\ E-mail: molitormekph.uni-mainz.de
}

\section{Kurt Aulenbacher}

Institut für Kernphysik, Johannes Gutenberg-Universität Mainz, Germany

E-mail: aulenbac@kph.uni-mainz.de

The P2 Experiment at the new Mainz Energy-recovering Superconducting Accelerator (MESA) aims at measuring the weak mixing angle $\sin ^{2} \theta_{\mathrm{W}}$ at low $\mathrm{Q}^{2}$ with high precision. Therefore the polarization of the incident electron beam has to be known with a very high accuracy $(<0.5 \%)$. A conventional Mott-polarimeter requires a lot of effort in calibration to be able to acieve this goal. A Double Scattering Polarimeter (DSP) promises to be able to meet these requirements with less effort.

XVIth International Workshop in Polarized Sources, Targets, and Polarimetry, PSTP2015,

14-18 September 2015,

Bochum, Germany

\footnotetext{
* Speaker.

${ }^{\dagger}$ Work sopported by DFG through Excellence-cluster PRISMA.
} 


\section{Introduction}

As a fundamental parameter of the Standard Model of particle physics, the weak mixing angle $\sin ^{2} \theta_{\mathrm{W}}$ has been measured with great precision at high $\mathrm{Q}^{2}$. In order to achieve equally precise measurements at lower $\mathrm{Q}^{2}$, the new Mainz Energy-recovering Superconducting Accelerator (MESA) is being constructed. At this new accelerator the $\mathrm{P} 2$ collaboration will measure the weak mixing angle in electron proton scattering with an average momentum transfer $\mathrm{Q}^{2}$ of $4.5 \cdot 10^{-3} \mathrm{GeV}^{2} / \mathrm{c}^{2}$ and aims for a precision of $0.13 \%$ in $\sin ^{2} \theta_{\mathrm{W}}$, which in turn requires to measure the parity violating asymmetry with an relative accuracy of $1.6 \%$. [1]

This level of precision requires the polarization of the incident electron to be known with an uncertainty of less than $0.5 \%$. In this case the error of the polarization measurement will cease to be a major limiting factor for the overall uncertainty. A crucial parameter in the determination of the polarization is the effective analyzing power $\left(S_{\text {eff }}\right)$ of the polarimeter, which can be determined in different ways. In this paper we discuss the possibility of elastic electron-nucleus scattering, which is frequently called "Mott-Scattering".

\section{Single Scattering Mott-Polarimeter}

In conventional Mott-Polarimeters the effective analyzing power is determined by measuring a left-right asymmetry $(A)$ of a polarized electron beam. For targets of finite thickness, multiple scattering leads to a dilution of the asymmetry-signal. Therefore, the asymmetry is recorded from a series of targets of various thicknesses. The asymmetry over thickness is then extrapolated to zero target thickness and this extrapolated value compared to the one that can be calculated from theory.

Tioukine et al. [2] discuss several error sources introduced by this method and conclude that it is very difficult to reduce these error sources much below $1 \%$ which does not meet the requirements just discussed.

\section{The Double Scattering Polarimeter}

In a DSP two identical targets are used to measure the effective analyzing power of the targets. Here an unpolarized beam is polarized at the first target. Electrons back-scattered under the angle $\Theta$, are collimated onto the second target and the asymmetry of the double scattered electrons is then measured at the same angle $\Theta$ with respect to the second target. With identical targets this results in two identical scattering processes and the effective analyzing power can be determined by

$$
S_{\text {eff }}=\sqrt{A}
$$

where $A$ is the experimentally observed counting asymmetry. 


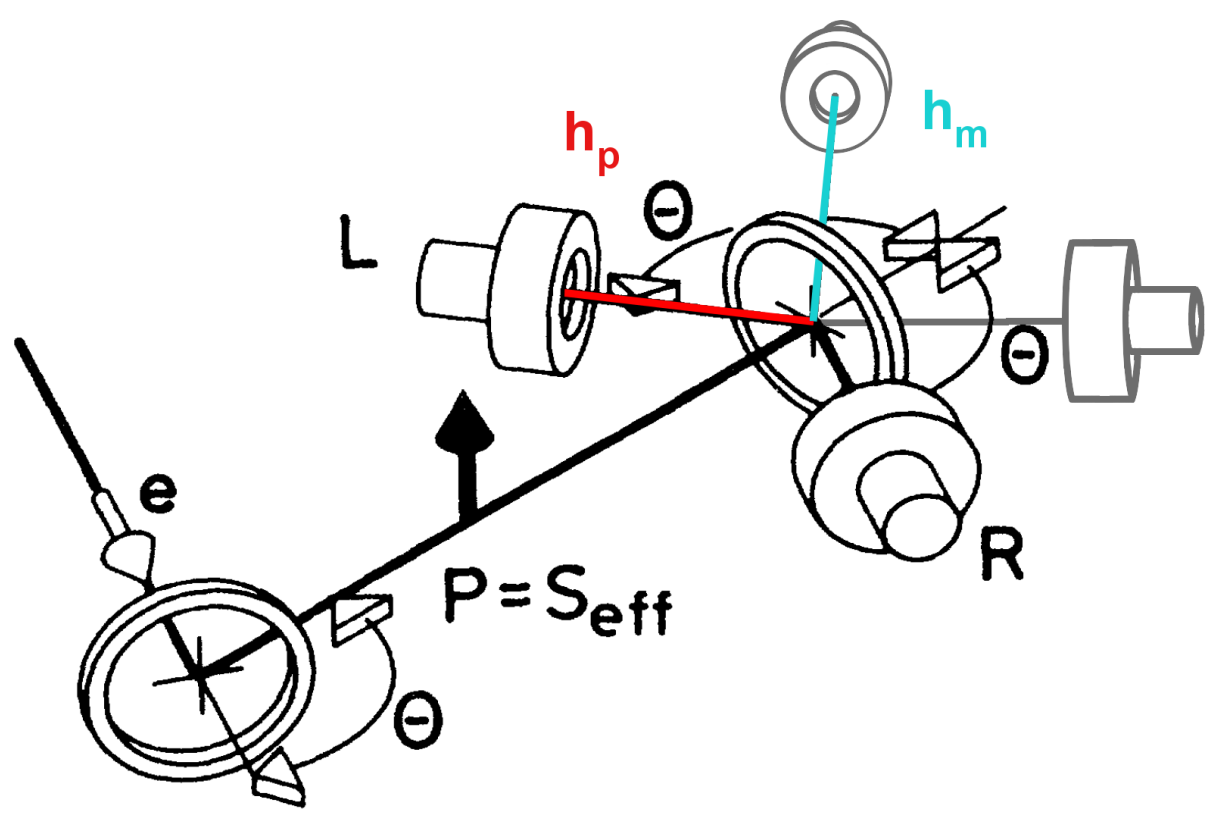

Figure 1: e: incident unpolarized beam; $P$ : polarized beam after first scattering; $h_{m} / h_{p}$ : distance from secondary target to the monitor/polarization counters. Adapted from [5]

Further cross-checks can be performed using a polarized primary beam [3]. By doing so and switching the polarization of the primary beam, the requirement for two identical targets can be dropped.

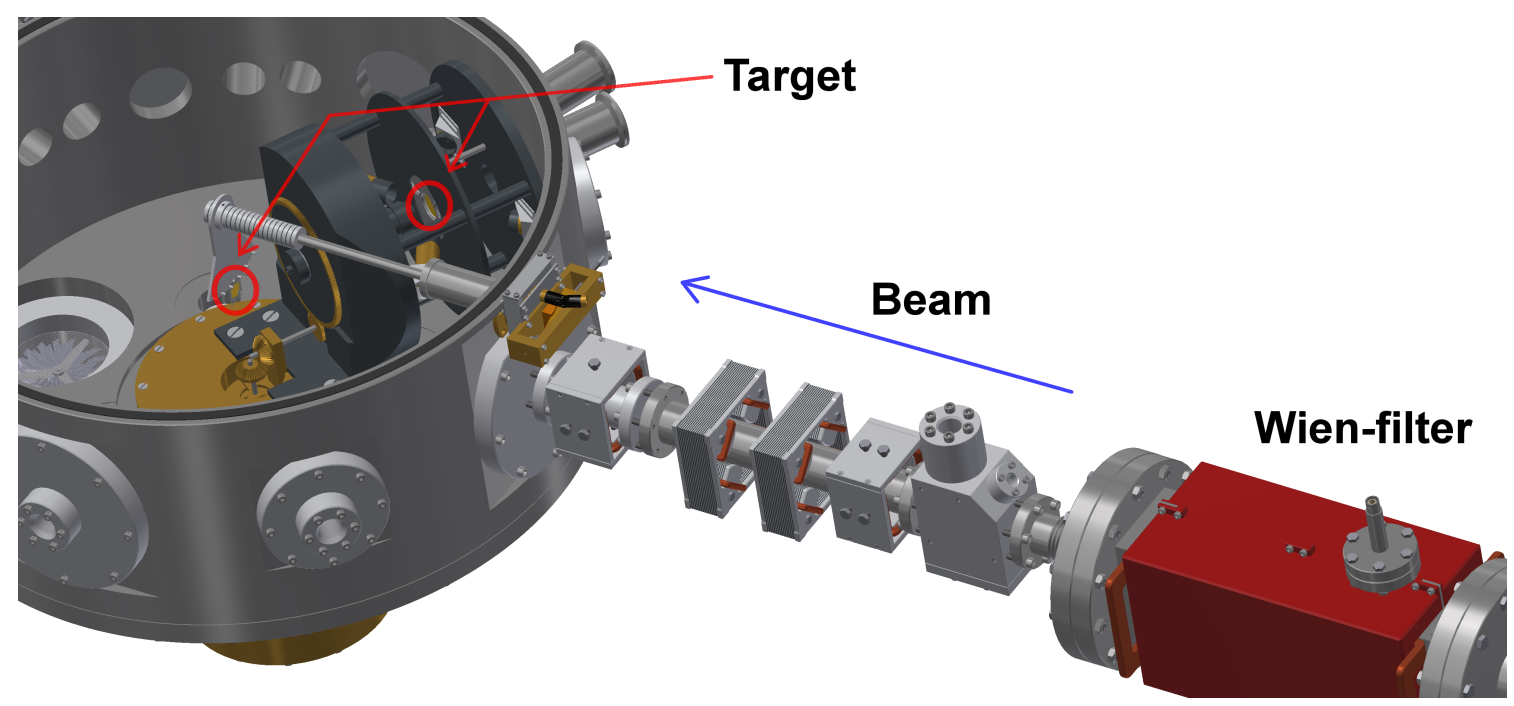

Figure 2: Computer drawing of the current DSP test setup with the Wien-filter (red)

The device is operated at $100 \mathrm{keV}$, which corresponds to the source energy of MESA. For the polarization measurement of an unknown electron-beam, a target with calibrated effective analyzing power is moved into the beam. The beam polarization is then given by

$$
P=A_{\exp } / S_{\text {eff }}
$$


This is an invasive measurement but the measurement time is very low due to the fact that only single scattering is necessary once the target has been calibrated. The accuracy in the determination of $S_{\text {eff }}$ is mainly limited by the control over instrumental asymmetries during the calibration.

\section{Instrumental Asymmetries}

In order to eliminate instrumental asymmetries, a second pair of counters, the monitor-counters, is placed under a forward angle of 45 degree, where the analyzing power is negligible. The ratio of the distances $h_{m} / h_{p}$ can be derived [4] from a measurement of the angle dependent intensity $(I(\Theta)$ ) of the scattered electrons in single scattering by

$$
\frac{h_{\mathrm{m}}}{h_{\mathrm{p}}}=\frac{\cos \Theta_{\mathrm{m}}-2\left[E\left(\Theta_{\mathrm{m}}\right)\right]^{-1} \sin \Theta_{\mathrm{m}}}{\cos \Theta_{\mathrm{p}}-2\left[E\left(\Theta_{\mathrm{p}}\right)\right]^{-1} \sin \Theta_{\mathrm{p}}}
$$

where $\mathrm{E}(\Theta)=(1 / \mathrm{I}(\Theta))(\partial \mathrm{I}(\Theta) / \partial \Theta)$ with $\mathrm{I}(\Theta)$ being the intensity of the beam after the first scattering. Afterwards the target used to determine $\mathrm{h}_{\mathrm{m}} / \mathrm{h}_{\mathrm{p}}$ is used as the secondary target in double scattering.

\section{First Measurements}

After careful adjustments of the counter-pairs, measurements to determine the effective analyzing power of a set of targets ( $74 \mu \mathrm{g}$ Gold on Formvar backing-foil) have been performed. The asymmetries of the single measurements for each counter-pair were put together in a histogram (see fig. 3) and both sets of detectors can be fitted with a gauss-distribution.

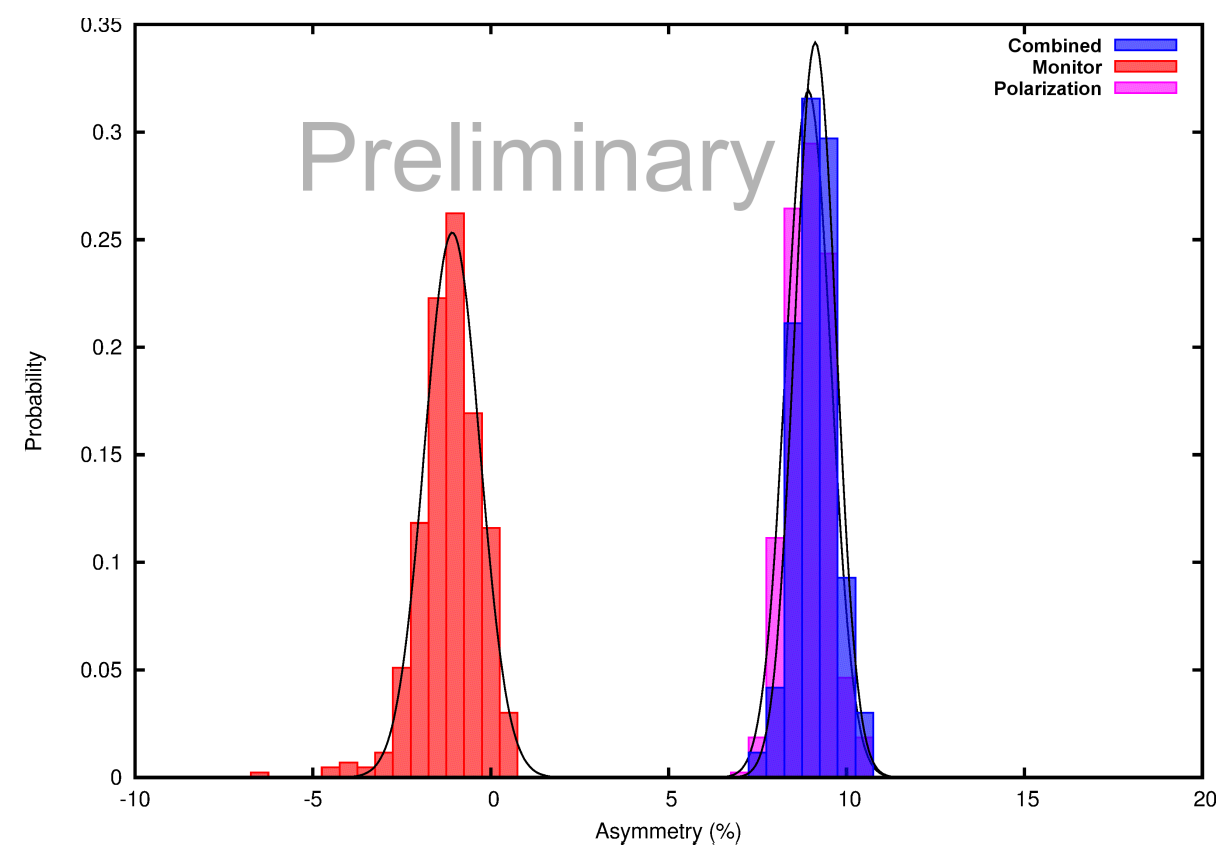

Figure 3: Histogram of 430 single measurements. Red: Asymmetry of monitor counters; Purple: Asymmetry of Polarization counters; Blue: Combined asymmetry of all four counters 
Correcting the asymmetry in the polarization-counters $\left(A_{p}\right)$ with the asymmetry in the monitorcounters $\left(A_{m}\right)$, we obtain the total asymmetry [4]

$$
\begin{gathered}
A=A_{\mathrm{p}}-c A_{\mathrm{m}} \\
c=E\left(\Theta_{\mathrm{p}}\right) / E\left(\Theta_{\mathrm{m}}\right)
\end{gathered}
$$

with a value of $A=9.384 \pm 0.005 \%$ from these measurements. This corresponds to an effective analyzing power of $S_{\text {eff }}=0.306 \pm 0.001$ (only taking statistical errors into account).

The determination of systematic errors is ongoing. An important part of this task will be to check for consistency of the results with polarized beam.

Since the backing foils can only withstand an increase of temperature towards approx. $380 \mathrm{~K}$ repeatedly, they limit the maximum beam current to $\approx 2 \mu \mathrm{A}$, therefore it takes 50 to 100 hours of beam time in order to gain enough statistic on one setup. Now, in December 2015, the DSP is being refitted with new targets, including $100 \mathrm{~nm}$ without backing foil, for comparison measurements. These new, pure gold, targets should allow for higher beam currents and rates in double scattering, reducing the data-taking time.

\section{Summary and Outlook}

The DSP promises an accuracy of $0.5 \%$ or even better, as required for the upcoming P2Experiment. Double scattering measurements have been performed and a first correction for instrumental asymmetries has been made. In order to determine the level of systematic uncertainty, additional measurements are required. These ongoing experiments with the DSP will include measurements with different targets and polarized beam to cross-check the data already obtained.

\section{References}

[1] N. Berger et al., Measuring the weak mixing angle with the P2 experiment at MESA, arXiv:1511.03934 [physics.ins-det]

[2] V. Tioukine, K. Aulenbacher and E. Riehn, A Mott polarimeter operating at MeV electron beam energies, Rev. Sci. Instrum. 82 (2011) 033303

[3] S. Meyer, T. Fischer, W.Blaschke, J. Kessler, Calibration of a Mott electron polarimeter: Comparison of different methods, Rev. Sci. Instrum. 64 (1993) 952

[4] A. Gellrich, K. Jost, J. Kessler, Elimination of instrumental asymmetries in electron polarization analysis, Rev. Sci. Instrum. 61 (1990) 3399

[5] A. Gellrich, J. Kessler, Precision measurement of the Sherman asymmetry function for electron scattering from gold, Phys. Rev. A 43 (1991) 204 